\title{
Does information entropy play a role in the expansion and acceleration of the Universe?
}

\author{
Biswajit Pandey ${ }^{\star}$ \\ Department of Physics, Visva-Bharati University, Santiniketan, Birbhum, 731235, India
}

12th March 2018

\begin{abstract}
We propose an interpretation of the expansion and acceleration of the Universe from an information theoretic perspective. We obtain the time evolution of the configuration entropy of the mass distribution in a static Universe and show that the process of gravitational instability leads to a rapid dissipation of configuration entropy during the growth of the density fluctuations making such a Universe entropically unfavourable. We find that in an expanding Universe, the configuration entropy rate is governed by the expansion rate of the Universe and the growth rate of density fluctuations. The configuration entropy rate becomes smaller but still remains negative in a matter dominated Universe and eventually becomes zero at some future time in a $\Lambda$ dominated Universe. The configuration entropy may have a connection to the dark energy and possibly plays a driving role in the current accelerating expansion of the Universe leading the Universe to its maximum entropy configuration.
\end{abstract}

Key words: methods: analytical - cosmology: theory - large scale structure of the Universe.

\section{INTRODUCTION}

The expanding Universe (Hubble 1929) and its current accelerating expansion (Riess et al. 1998; Perlmutter et al. 1999a) are the two milestone discoveries in observational cosmology. In the current standard model of cosmology, the accelerating expansion of the Universe is explained by an all permeating hypothetical form of energy known as the dark energy. Independent observations from high redshift supernova (Riess et al. 1998; Perlmutter et al. 1999a), cosmic microwave background radiation (Sherwin et al. 2011; Hinshaw et al. 2013; Planck Collaboration et al. 2016a), gravitational lensing (Sarbu et al. 2001; Weinberg \& Kamionkowski 2003; Cao et al. 2012) and large scale structures (Perlmutter et al. 1999b; Blanchard et al. 2006; Pavlov et al. 2014) suggest that the dark energy constitutes almost $\sim 70 \%$ of the total energy density of the present day Universe. However the exact nature of the dark energy is still unknown and remains matters of speculation. The $\Lambda \mathrm{CDM}$ model is currently the most successful model in explaining a wide range of cosmological observations. In the $\Lambda \mathrm{CDM}$ model, one may choose to identify the cosmological constant as the dark energy. However this leads to a discrepancy by an embarrassing 120 orders of magnitude between the tiny observed value of the cosmological constant and its large theoretically predicted value. Various alternatives to dark energy for explaining the observed accelerated expansion has been proposed since its inception. Buchert (2000) used the backreaction mechanism resulting from the non-commutativity

^ E-mail: biswap@visva-bharati.ac.in of time evolution and spatial averaging in a perturbed Universe to explain the accelerating expansion. The presence of a large local void can also mimic an apparent acceleration of expansion (Tomita 2001; Hunt \& Sarkar 2010). The entropic force arising from the information storage on the horizon surface screen can also produce an acceleration (Easson et al. 2011). Some more recent works suggest that the non-zero vacuum energy density of the Universe is related to the finite amount of accessible information stored in the spacetime (Padmanabhan 2016; Padmanabhan \& Padmanabhan 2017). At present, understanding the dark energy and the cosmic acceleration remains one of the most profound puzzles in cosmology and science in general.

In the present work, we consider the information entropy of the Universe together with the other sources of entropy generation and apply the maximum entropy production principle to understand the expansion and acceleration of the Universe. The present entropy budget of the Universe is made up of several components. One can calculate the contributions from the CMB photons, stellar photons, neutrinos and gravitons to the total entropy (Egan \& Lineweaver 2010). The entropy of these relativistic particles do not change during the expansion of the Universe. The entropy of the baryonic matters in stars, interstellar medium (ISM) and the intergalactic medium (IGM) can be estimated from the Sackur-Tetrode equation (Basu \& Lynden-Bell 1990; Egan \& Lineweaver 2010) which gives the entropy per baryon. The entropy in the baryonic component decreases with time as it becomes more structured during the evolution of the Universe. The growth of the Stellar Black Holes $(\mathrm{SBH})$ and the Supermassive Black Holes (SMBH) are also known 
as two efficient sources of entropy in the Universe. The entropy of a Schwarzschild black hole is well known (Bekenstein 1973; Hawking 1976) which can be integrated over the SBH and SMBH mass functions to estimate their contributions to the total entropy of the Universe (Penrose 2004; Frampton 2009; Egan \& Lineweaver 2010). Further, most of the mass in the Universe is in the form of dark matter. Calculating the entropy of the dark matter is tricky as its exact nature is unknown. However, one can calculate it assuming that the dark matter is weakly interacting massive particle (WIMP) (Egan \& Lineweaver 2010). Besides these contributions, the total entropy within the Cosmic Event Horizon (CEH) must also include an additional contribution from CEH itself which can be calculated using the formula provided by Gibbons \& Hawking (1977). The entropy of the horizon largely dominates the other sources of entropy in the Universe (Egan \& Lineweaver 2010).

Finally, the mass distribution in the present Universe is highly organized into complex hierarchical patterns. The galaxies are found to be distributed in a complex network of clusters and filaments surrounded by voids. This complex network is often referred as the cosmic web (Bond et al. 1996). The cosmic web emerges naturally by the process of gravitational instability which amplifies the tiny density fluctuations seeded in the early Universe. We argue that one should also take into consideration the change in the entropy of the Universe associated with its evolution from a nearly smooth distribution to a highly organized complex structure. We define the configuration entropy of the mass distribution based on the idea of information entropy (Shannon 1948) and study its time evolution in the linear regime using the linear perturbation theory. If the second law of thermodynamics applies to our Universe as a whole then the sum of all the entropies in the Universe must increase with time since the big bang. We study how the time evolution of the configuration entropy affects the evolution of the total entropy of the Universe which may in turn influence the dynamics of the Universe on large scales.

The connection between the cosmic accelerated expansion and the second law of thermodynamics has been studied extensively (Radicella \& Pavón 2012; Pavón \& Radicella 2013; Mimoso \& Pavón 2013; Pavón 2014; Ferreira \& Pavón 2016). The accelerated expansion of the Universe is consistent with the second law of thermodynamics and it could have been anticipated on thermodynamic grounds before its discovery from the observations of high redshift supernova (Radicella \& Pavón 2012; Pavón 2014). Based on the Hubble expansion history, Pavón (2014) suggested that the entropy of the Universe tends to some maximum value. Interestingly different cosmological models such as nonsingular bouncing Universes, modified gravity theories and the Universes whose expansion is dominated by matter or by phantom fields do not tend to a state of maximum entropy whereas the ACDM model does (Radicella \& Pavón 2012; Mimoso \& Pavón 2013; Ferreira \& Pavón 2016).

The information entropy has been used earlier in cosmology to study various issues like homogeneity (Hosoya et al. 2004; Pandey 2013), isotropy (Pandey 2016a), complexity (Vazza 2017), bias and non-Gaussianity (Pandey 2016b), information entanglement (Czinner 2016), dark energy from entanglement (Cappzziello \& Luongo 2013, 2017) and the large scale environmental dependence of galaxy properties (Pandey \& Sarkar 2017). In the present work, we use information entropy to understand the expansion and acceleration of the Universe.

A brief outline of the paper follows. In section 2 we describe the configuration entropy and its time evolution in a static and an expanding Universe. We discuss the possible roles of the config- uration entropy in the observed expansion and acceleration of the Universe and present our conclusions in section 3.

\section{CONFIGURATION ENTROPY AND ITS TIME EVOLUTION}

The continuity equation in a fluid is,

$$
\frac{\partial \rho}{\partial t}+\nabla \cdot(\rho \vec{v})=0
$$

where $\rho$ is the fluid density and $\vec{v}$ is the flow velocity. We define the configuration entropy of the fluid as,

$$
S_{C}(t)=-\iiint \rho \log \rho d x d y d z
$$

where the entire space occupied by the fluid is divided into a finite number of elements with volume $d V=d x d y d z$.

Multiplying Equation 1 by $(1+\log \rho)$ and integrating over the whole space we get,

$$
\frac{d S_{c}}{d t}-\int \nabla \cdot(\rho \log \rho \vec{v}) d V-\int \rho \nabla \cdot \vec{v} d V=0
$$

We consider a sufficiently large volume of the Universe. The second term in the Equation 3 can be expressed as a surface integral which vanishes at the boundary. So we finally have,

$$
\frac{d S_{c}}{d t}=\int \rho \nabla \cdot \vec{v} d V
$$

A similar relation has been obtained by Liang \& Kleeman (2005) in the context of information transfer between dynamical system components.

\subsection{CONFIGURATION ENTROPY IN A STATIC UNIVERSE}

We consider a self-gravitating fluid with small fluctuations in its density, pressure and potential in a static Universe. This leads to the well known Jeans instability in a static Universe. In this case the linearized continuity equation becomes,

$$
\nabla \cdot \vec{v}=-\frac{\partial \delta}{\partial t}
$$

where $\delta(\vec{r}, t)=\frac{\rho(\vec{r}, t)-\bar{\rho}}{\bar{\rho}}$ is the density contrast and $\bar{\rho}$ is the mean density.

Substituting $\nabla \cdot \vec{v}$ in Equation 4 gives,

$$
\frac{d S_{C}}{d t}=-\int \bar{\rho}(1+\delta) \frac{\partial \delta}{\partial t} d V
$$

As of now we have only used the equation of continuity which ensures mass conservation during the fluid flow. Combining the Euler's equation and Poisson's equation with the continuity equation yields the equation governing the growth of density perturbations $\delta(\vec{r}, t)$ in a static Universe. The solution of this equation tells us that the density perturbations larger than the Jeans length will grow exponentially as $\delta(\vec{r}, t)=\delta(\vec{r}) \exp (\sqrt{4 \pi G \bar{\rho}} t)$. Substituting this in Equation 6 and simplifying we get,

$$
\frac{d S_{c}}{d t}=-\bar{\rho} \sqrt{4 \pi G \bar{\rho}} \exp (2 \sqrt{4 \pi G \bar{\rho}} t) \int \delta^{2}(\vec{r}) d V
$$

The integral in Equation 7 is related to the variance of the fluctuations and is a positive quantity. This suggests that the configuration entropy rate of a self-gravitating fluid in a static Universe is negative and its magnitude blows up exponentially with time. 


\subsection{CONFIGURATION ENTROPY IN AN EXPANDING UNIVERSE}

Now we consider the growth of the density perturbations in a self gravitating fluid in an expanding Universe. The continuity equation in the comoving co-ordinate is,

$$
\frac{\partial \rho}{\partial t}+3 \frac{\dot{a}}{a} \rho+\frac{1}{a} \nabla \cdot(\rho \vec{v})=0
$$

where $a$ is the cosmological scale factor and $\vec{v}$ is the peculiar velocity.

Multiplying Equation 8 by $(1+\log \rho)$ and integrating over the whole space gives,

$$
\frac{d S_{c}(t)}{d t}+3 H(t) S_{c}(t)-\frac{1}{a} \int \rho(3 \dot{a}+\nabla \cdot \vec{v}) d V=0
$$

where $H(t)=\frac{\dot{a}}{a}$ is the Hubble parameter. We express the third term in the left hand side of Equation 9 as some function of time $F(t)=\frac{1}{a} \int \rho(3 \dot{a}+\nabla \cdot \vec{v}) d V$. One may note that $F(t)$ can be positive, negative or zero for the different parts of the fluid volume. $F(t)$ will be zero when the divergence of the peculiar flow is cancelled by the divergence of the Hubble flow, positive when the Hubble flow dominates over the peculiar flow and negative otherwise.

We now have an ordinary differential equation,

$$
\frac{d S_{c}(t)}{d t}+3 H(t) S_{C}(t)-F(t)=0
$$

The solution of Equation 10 gives the evolution of the configuration entropy in an expanding Universe which is given by,

$$
\begin{gathered}
S_{C}(t)=\exp \left(-3 \int_{t_{0}}^{t} H\left(t^{\prime}\right) d t^{\prime}\right)\left[S_{C}\left(t_{0}\right)\right. \\
\left.+\int_{t_{0}}^{t} F\left(t^{\prime \prime}\right) \exp \left(3 \int_{t_{0}}^{t^{\prime \prime}} H\left(t^{\prime}\right) d t^{\prime}\right) d t^{\prime \prime}\right]
\end{gathered}
$$

where $S_{C}\left(t_{0}\right)$ is the configuration entropy at time $t_{0}$. Noticeably, the magnitude of the entropy rate becomes smaller in an expanding Universe as compared to a static Universe and it would depend on the form of the function $F(t)$. We can express $F(t)$ as,

$$
\begin{aligned}
F(t) & =3 M H(t)+\frac{1}{a} \int \rho(\vec{x}, t) \nabla \cdot \vec{v} d V \\
& =F_{1}(t)+F_{2}(t)
\end{aligned}
$$

where $\vec{x}$ is the comoving co-ordinate and $M=\int \rho(\vec{x}, t) d V=$ $\int \bar{\rho}(1+\delta(\vec{x}, t)) d V$ is the total mass enclosed inside the comoving volume $V$.

One can simplify $F(t)$ further using the linear perturbation theory. The linearized continuity equation in the comoving co-ordinate becomes,

$$
\nabla \cdot \vec{v}=-a \frac{\partial \delta}{\partial t}
$$

In the linear regime, the shape of the density fluctuations remain frozen in comoving co-ordinates and their amplitude grows as $\delta(\vec{x}, t)=D(t) \delta(\vec{x})$ where $D(t)$ is the growing mode of density perturbations. The second term in Equation 12 can be expressed as,

$$
\begin{aligned}
F_{2}(t) & =-\bar{\rho} \dot{D}(t) D(t) \int \delta^{2}(\vec{x}) d V \\
& =-\bar{\rho} H(t) f D^{2}(t) \int \delta^{2}(\vec{x}) d V
\end{aligned}
$$

where $f=\frac{d \ln D}{d \ln a}$ is the logarithmic derivative of the growing mode with respect to the scale factor.

\subsection{CONFIGURATION ENTROPY RATE IN A MATTER DOMINATED UNIVERSE}

We use the linear perturbation theory to calculate the configuration entropy rate in the linear regime in a matter dominated expanding Universe. The configuration entropy rate depends on the form of the function $F(t)$ in Equation 12. In case of a matter dominated Universe, we have $H(t) \propto \frac{1}{t}, D(t) \propto a(t)$ and $a(t) \propto t^{\frac{2}{3}}$. Consequently $F_{1}(t)$ will scale as $t^{-1}$ and $F_{2}(t)$ will scale as $t^{\frac{1}{3}}$. Since $F_{2}(t)$ is negative so $F(t)$ will become negative after large $t$.

This suggests that the configuration entropy rate decreases significantly in a matter dominated Universe but still remains negative after a long time. This is related to the ongoing growth of perturbations on all scales in a matter dominated Universe.

\subsection{CONFIGURATION ENTROPY RATE IN A $\Lambda$ DOMINATED UNIVERSE}

Now we analyze the situation in a $\Lambda$ dominated Universe. In a $\Lambda$ dominated Universe, $H(t)=$ constant, $D(t)=$ constant and $a(t) \propto \exp (H t)$. Thus $F_{1}(t)$ becomes a constant and $F_{2}(t)=0$. As a result the first term in Equation 11 is exponentially damped and the second term converges to a constant value. When $S_{C}(t)$ reaches a constant value, the configuration entropy rate $\frac{d S_{c}}{d t}=0$.

This suggests that the configuration entropy converges to a constant value in the linear regime in a $\Lambda$ dominated Universe and there is no longer any increase or decrease in the configuration entropy on large scales. The density perturbations stop growing on large scales in an $\Lambda$ dominated Universe leading to $\frac{d S_{c}}{d t}=0$ which maximizes the entropy production.

\section{DISCUSSION AND CONJECTURE}

We find that the configuration entropy decreases when the Universe self-organizes itself into complex patterns from a nearly homogeneous and isotropic fluid with tiny fluctuations seeded in the early Universe. If the second law of thermodynamics applies to the Universe as a whole then the total entropy of the Universe must increase with time. The total entropy of the Universe is distributed into several components. Let us write the total entropy inside a significantly large comoving volume in the Universe as $S_{T}(t)=S_{\text {other }}(t)+S_{C}(t)$ where $S_{\text {other }}(t)$ is the sum of all other entropies in that volume except the configuration entropy $S_{C}(t)$. The volume is bounded by a closed comoving surface and the large scale homogeneity and isotropy allows us to treat this volume as an isolated system as there are no net flows across the neighboring volumes. Consequently, we can claim that $\frac{d S_{T}(t)}{d t}=\frac{d S_{\text {other }}(t)}{d t}+\frac{d S_{c}(t)}{d t}>0$ inside that volume. This applies to all such large volumes and the Universe as a whole. The Equation 7 shows that $\frac{d S_{c}(t)}{d t}<0$ and the magnitude of the dissipation rate of the configuration entropy grows exponentially with time when the structure formation continues in a static Universe. This implies that in the long run, the negative configuration entropy rate will eventually dominate the total entropy rate leading to a negative total entropy rate $\frac{d S_{T}(t)}{d t}<0$ in that volume. This is particularly important when the other entropy generation processes are not as efficient as the dissipation of the configuration entropy in that volume. None of the known entropy generation mechanisms in the Universe can produce entropy at such a faster rate. So a static Universe is not entropically favoured in the presence of density fluctuations. 
On the other hand, the density perturbations no longer grow exponentially in an expanding Universe. Instead they grow as a power law because gravity has to work a lot harder against the Hubble drag to form structures in such a Universe. The Equation 11 show that the dissipation rate of the configuration entropy becomes smaller in an expanding Universe. The configuration entropy rate depends on the rate of expansion of the Universe and the growth rate of perturbations inside it. Clearly, the configuration entropy rate would depend on the cosmological model. In a matter dominated expanding Universe, the configuration entropy rate becomes negative after a large time. The Universe is slowed down and the growth rate of structures is enhanced in such model. Consequently, the configuration entropy would dissipate at a higher rate under such conditions. If the other entropy generation processes are not as efficient as the dissipation, it may again lead to an unphysical situation such as $\frac{d S_{T}(t)}{d t}<0$. The validity of the second law of thermodynamics demands that the dissipation rate of the configuration entropy has to be always less than the collective entropy generation rates from all the other sources.

The configuration entropy continues to dissipate in a matter dominated Universe because the fluctuations on larger scales continue to grow. The only way to control the dissipation is to suppress the growth of structures by increasing the Hubble drag. This is exactly what we see in a Universe dominated by the cosmological constant. In a lambda dominated Universe, the configuration entropy converges to a constant value after the growth of structures on linear scales are shut off. The accelerated expansion of the Universe damps out the growth of structures on linear scales and leads to an ideal situation where the dissipation of the configuration entropy cease to exist. Here we would like to mention that our analysis is only limited to the linear regime and hence misses out the dissipation of the configuration entropy due to the growth of non-linear structures.

The dissipation of the configuration entropy is also supported by the fact that the information entropy decreases when a Gaussian distribution turns in to a non-Gaussian distribution. The Gaussian distribution has the maximum information entropy among all other distributions with a specified variance. It is well known from the $\mathrm{CMB}$ observations that the primordial fluctuations from the early Universe were highly Gaussian (Planck Collaboration et al. 2014, 2016b) whereas the present day mass distribution in the Universe is highly non-Gaussian. The large scale structures in the Universe emerge from the growth of these fluctuations via the process of gravitational instability which developes non-Gaussianity in the distribution leading to a dissipation of the information entropy.

Admittedly, the analysis presented in this work does not yet provide an alternative to the dark energy. But it certainly points out to some interesting links between the dark energy and the configuration entropy of the Universe. Based on our observations, here we propose a conjecture without providing a rigorous proof. The homogeneity and isotropy is the most preferred and natural state of the Universe as it corresponds to the state with maximum information. The fact that our Universe is represented by the homogeneous and isotropic Friedman-Lemaitre-Robertson-Walker (FLRW) metric on large scales is possibly an evidence that the Universe maintains its status quo despite the growth of perturbations in it. So the cosmological principle is not merely a choice but a requirement. The Early Universe was homogeneous and isotropic to a very high degree of precision and thus possessed higher information entropy on all scales. The Universe still retains maximum amount of information beyond the scale of homogeneity and isotropy. But the mass distribution on small scales is highly inhomogeneous and anisotropic due to the formation of structures by gravitational clustering. Any deviation from homogeneity and isotropy acts as a sink of information requiring a balance of entropy. Each inhomogeneous and anisotropic patch of the Universe below the scale of homogeneity and isotropy may thus experience an entropic force resulting into an acceleration which ensures the maximum entropy production principle. Besides the different mass-energy densities in the Universe, the information entropy may also take a decisive role in determining the large scale dynamics of the Universe. It is interesting to note that if this is true then there exists a natural explanation to the coincidence problem which asks why the present densities of matter and dark energy happen to be the same order of magnitude. If the accelerated expansion of the Universe results from its response to the dissipation of the configuration entropy in a matter dominated Universe then they are expected to be of the same order of magnitude.

\section{ACKNOWLEDGEMENT}

I sincerely thank an anonymous reviewer for the useful comments and suggestions. The author would like to acknowledge financial support from the SERB, DST, Government of India through the project EMR/2015/001037. The author would also like to acknowledge IUCAA, Pune and CTS, IIT, Kharagpur for providing support through associateship and visitors programme respectively.

\section{References}

Basu, B., \& Lynden-Bell, D. 1990, QJRAS, 31, 359

Bekenstein, J. D. 1973, Physical Review D, 7, 2333

Blanchard, A., Douspis, M., Rowan-Robinson, M., \& Sarkar, S. 2006, A\&A, 449,925

Bond J. R., Kofman L., Pogosyan D. 1996, Nature, 380, 603

Buchert, T. 2000, General Relativity and Gravitation, 32, 105

Cao, S., Covone, G., \& Zhu, Z.-H. 2012, ApJ, 755, 31

Capozziello, S. \& Luongo, O. 2013 International Journal of Theoretical Physics, 52, 2698

Capozziello, S. \& Luongo, O. 2017 International Journal of Theoretical Physics, arXiv: 1704.00195

Czinner, V. G. \& F. C. Mena 2016 Physics Letters B, 758, 9

Easson, D. A., Frampton, P. H., \& Smoot, G. F. 2011, Physics Letters B, 696, 273

Egan, C. A., \& Lineweaver, C. H. 2010, ApJ, 710, 1825

Ferreira, P. C., \& Pavón, D. 2016, European Physical Journal C, 76, 37

Frampton, P. H. 2009, JCAP, 10, 016

Gibbons, G. W., \& Hawking, S. W. 1977, Physical Review D, 15, 2738

Hawking, S. W. 1976, Physical Review D, 13, 191

Hinshaw, G., Larson, D., Komatsu, E., et al. 2013, ApJS, 208, 19

Hosoya, A., Buchert, T., \& Morita, M. 2004, Physical Review Letters, 92, 141302

Hubble, E. 1929, Proceedings of the National Academy of Sciences of the U.S.A., 15, 168

Hunt, P. \& Sarkar, S. 2010, MNRAS, 401, 547

Liang, X. San, \& Kleeman, R. 2005, Physical Review Letters, 95(24), 244101

Mimoso, J. P., \& Pavón, D. 2013, Physical Review D, 87, 047302

Padmanabhan, T. 2016, arXiv:1611.03505

Padmanabhan, T., \& Padmanabhan, H. 2017, arXiv:1703.06144

Pandey, B. 2013, MNRAS, 430, 3376

Pandey, B. 2016, MNRAS, 462, 1630

Pandey, B. 2016, MNRAS, 463, 4239

Pandey, B., \& Sarkar, S. 2017, MNRAS, 467, L6

Pavlov, A., Farooq, O., \& Ratra, B. 2014, Physical Review D, 90, 023006

Pavón, D., \& Radicella, N. 2013, General Relativity and Gravitation, 45, 63 
Pavón, D. 2014, International Journal of Geometric Methods in Modern Physics, , 11, 1460007

Penrose, R. 2004, The road to reality : a complete guide to the laws of the universe, by Roger Penrose. London: Jonathan Cape, 2004,

Perlmutter, S., Aldering, G., Goldhaber, G., et al. 1999, ApJ, 517, 565

Perlmutter, S., Turner, M. S., \& White, M. 1999, Physical Review Letters, 83,670

Planck Collaboration, Ade, P. A. R., Aghanim, N., et al. 2014, A\&A, 571, A24

Planck Collaboration, Ade, P. A. R., Aghanim, N., et al. 2016, A\&A, 594, A13

Planck Collaboration, Ade, P. A. R., Aghanim, N., et al. 2016, A\&A, 594, A17

Radicella, N., \& Pavón, D. 2012, General Relativity and Gravitation, 44, 685

Riess, A. G., Filippenko, A. V., Challis, P., et al. 1998, AJ, 116, 1009

Sarbu, N., Rusin, D., \& Ma, C.-P. 2001, ApJ Letters, 561, L147

Shannon, C. E. 1948, Bell System Technical Journal, 27, 379-423, 623-656

Sherwin, B. D., Dunkley, J., Das, S., et al. 2011, Physical Review Letters, 107,021302

Tomita, K. 2001, MNRAS, 326, 287

Vazza, F. 2017, MNRAS, 465, 4942

Weinberg, N. N., \& Kamionkowski, M. 2003, MNRAS, 341, 251

This paper has been typeset from a $\mathrm{T}_{\mathrm{E}} \mathrm{X} / \mathrm{LT} \mathrm{E} \mathrm{X}$ file prepared by the author. 